\title{
Research on the effect of Financial agglomeration to Technical efficiency of Chinese New High-tech Industry
}

\author{
ZHANG Caijiang ${ }^{1, a}$, LI Yifang 2,b,*
}

1 South China University Of Technology, Guangzhou, China

2 South China University Of Technology, Guangzhou, China

acaijiangzhang@126.com, b13668942637@163.com

${ }^{*}$ Corresponding author

Keywords: Financial agglomeration, New High-tech Industry, Malmquist index, Panel model.

\begin{abstract}
This paper calculated the innovation index of new High-tech industry through the method of DEA-Malmquist by using the data of 28 provinces during 2008-2013. In the base of this index , it studies the relationship of financial agglomeration and technological innovation. Results shows that bank agglomeration and securities agglomeration can promote innovation significantly excluding insurance. In terms of the effect of EFF and TECH, it shows that the main influence comes from TECH not EFF.
\end{abstract}

\section{金融集聚的创新驱动效应分析：对我国高技术产业的实证研究

\author{
张彩江 ${ }^{1, a}$, 李艺芳 $2, b,{ }^{*}$ \\ 1 华南理工大学经济与贸易学院, 广州, 广东, 中国 \\ 2 华南理工大学经济与贸易学院, 广州, 广东, 中国 \\ acaijiangzhang@126.com, b13668942637@163.com
}

通讯作者

关键词：金融集聚，高技术产业，Malmquist 指数，面板模型

中文摘要: 本文利用 2008-2013 年 28 个省际面板数据, 通过 DEA-Malmquist 指数方法测算 出我国各地区高技术产业创新效率的基础上, 探讨金融集聚（银行、证券、保险）及其区域 内创新效率间的本质关系, 并进一步从创新效率指数的分解指数 (EFF、TECH) 出发考察金 融集聚的具体促进效应, 结果发现, 在总创新效率方面, 除了保险业, 银行业、证券业两者 的集聚能显著地促进高技术产业创新效率的提高, 在分解指数方面, 这种集聚促进效应主要 作用于 TECH（技术进步效应），而不是 EFF（技术效率效应）。

\section{1. 引言}

创新是一个国家进步的动力来源, 而高技术产业集合了高科技含量产出、低能耗、高环 保的优良特性, 是技术创新的重要代表产业, 也是新经济的重要组成部分, 已经成为经济增 长的先导性产业。高技术产业的技术创新能力不仅是推动其发展的核心动力, 也对我国转变 经济增长方式、实现创新型国家的目标有着举足轻重的作用。金融是现代经济的血液, 也是 促进自主创新效率的重要变量，科技与金融结合也逐渐受到越来越多的重视。 


\section{2. 文献概述}

金融与创新的关系，著名创新经济学家熊彼特（Schumpeter） [1]早在 1912 年[1]就已首次 正式指出了金融促进技术创新, 并对作用途径进行了详细的阐述。此后围绕这一主题, 西方 学者开始展开一系列的研究, Hicks (1969) [1]通过阐述低流动性的资本对技术创新的重要性来 强调金融市场对企业技术创新的促进作用。而 Romer(1990) [1]从经济增长模型的构建角度出 发, 改变技术创新比率是金融体系的功能影响经济的稳定增长的重要途径。Saint-Paul (1992) ${ }^{[1]}$ 则认为金融市场保障了高风险、高报酬兼并的创新活动，从而促进了技术的进步。Luigi Benfratello 等 (2008) [1]通过对 20 世纪 90 年代意大利企业创新情况的调查实际分析, 探讨了 地方银行发展情况 (以银行分支机构密度为代理变量) 对企业创新行为的影响。Brown 等

（2009） [1]使用 1990-2004 年的 1347 个公开贸易的美国七个高科技行业企业的面板数据进 行实证研究，证明了金融、创新和经济增长三者之间有显著的联系。

而在国内，邓楠（1999）[1]、巴曙松（2000） [1]首先理论阐述了不同发展阶段的高技术产 业所存在的资金需求及风险投资体系, 并在此基础上提出了不同金融机构在高技术产业发展 中的重要地位, 马晓霞(2006) [1]以我国高技术企业普遍存在融资难的角度出发, 提出有效的 金融支持体系对提高我国高技术产业的创新能力的重要性, 黄德春等（2011） ${ }^{[1]}$ 实证得出了 金融发展规模和金融效率两者对中国高新技术产业的技术创新有明显的促进作用，且金融规 模存在门槛效应, 张长征等（2012） [1]通过加入高技术产业集聚效应进行实证分析, 得出金 融市场能推动其产业集聚和提高创新效率, 并且表明我国金融市场效率低的现状已成为阻碍 高新技术产业创新效率的提高的主要原因, 马彦新（2012） ${ }^{[1]}$ 在细分金融市场的前提下，得 出当前我国金融的深化、保险市场的发展和股票发展对提高高技术产业自主创新水平有显著 的正相关。

从已有文献研究可以看出技术创新水平的提高就意味着要有效率足够高、规模足够大的 金融支持, 而近年来, 随着金融资源在区域间加速流动, 当代金融业深度发展过程中显示出 来越来越明显的集聚趋势, 金融集聚已是不可忽视的显著现象, 刘军等(2007) [1]、丁艺等 （2009） [1]系统阐述了金融集聚通过外部规模经济效益、加速技术进步效益、创新效益、自 我强化机制效益等途径影响经济增长, 指出金融集聚区有利的知识资源、创新环境及服务水 平都会促进技术进步和提高创新效率, 并在此基础上进而促进经济增长和优化产业结构, 并 且石沛等 (2011) [1]、孙晶等 (2012) [1]、潘辉等 (2013) [1]等对此进行了实证验证。但根据 已有文献可以发现, 大多研究金融集聚的经济增长效应、产业升级效应, 但其产生的技术创 新效应研究却十分缺乏, 因此本文以高技术产业创新效率为研究对象, 利用 2008-2013 年间 省级层面的面板数据, 通过 DEA-Malmquist 指数方法测算出我国各地区高技术产业自主创 新效率及其构成指数的基础上，试图探讨金融集聚与我国高技术产业自主创新效率间的本质 关系, 并从创新效率指数的分解指数出发, 进一步考察金融集聚对我国高技术产业创新效率 增长效应的驱动来源。

\section{3. 计量模型、变量与数据}

\section{1 计量模型}

为了考察金融集聚对我国高技术产业创新效率的影响，本文以银行业集聚、证券业集聚 以及保险业集聚来具体代表金融集聚水平, 并参考大多数创新产出研究的模型构造方法, 采 用如下的柯布一道格拉斯函数形式的创新产出模型:

$$
Y_{i t}=A_{i t}\left(b_{i t}, s_{i t}, i_{i t}\right) f\left(k_{i t}, l_{i t}\right)=A_{i t}\left(b_{i t}, s_{i t}, i_{i t}\right) k_{i t}^{\alpha} l_{i t}^{\beta}
$$

其中， $Y_{i t}$ 代表创新产出， $k_{i t}$ 代表研发资本投入， $l_{i t}$ 代表研发人力投入，创新效率系数 $A_{i t}$ 是 $b_{i t}$ (银行业集聚水平)、 $s_{i t}$ (证券业集聚水平)、 $i_{i t}$ (保险业集聚水平) 的函数, $\alpha 、 \beta$ 分 别为 $k_{i t} 、 l_{i t}$ 对应的弹性系数, 下标 $i 、 t$ 分别表示省份和时间。 
此外，除了金融集聚因素外，研究经费投入强度、人力资本水平等其他因素均会影响影 响创新效率, 所以创新效率 $A_{i t}$ 设为:

$$
A_{i t}\left(b_{i t}, s_{i t}, i_{i t}\right)=Y_{i t} / f\left(k_{i t}, l_{i t}\right)=u_{i t} c_{i t}^{\eta} b_{i t}^{\delta} s_{i t}^{\phi} i_{i t}^{\gamma}
$$

其中 $u_{i t}$ 是不可观测的影响因素， $c_{i t}$ 为影响高技术产业创新效率的其他控制变量， $\eta 、 \delta$ 、 $\phi 、 \gamma$ 分别为 $c_{i t} 、 b_{i t} 、 s_{i t} 、 i_{i t}$ 对应的弹性系数。将上式两边取对数并进行整理得出本文的基 本计量模型为如下:

$$
\ln A_{i t}=a+\eta \ln c_{i t}+\delta \ln b_{i t}+\phi \ln s_{i t}+\gamma \ln i_{i t}+v_{i t}
$$

其中 $v_{i t}=\ln u_{i t}$ 。

\section{2 变量说明}

\subsection{1 被解释变量总创新效率及其分解的测算}

目前国内外学者常用的测量效率的方法主要有基于数据包络分析(Data Envelopment Analysis, 简称 DEA) 方法下的 Malmquist 指数及其分解 (包括技术效率变化和技术进步变化), 这种方法不需要特定的生产函数和无效率项的分布假设, 避免了人为设定生产函数所带来的 估计误差, 不需要相关的价格信息, 尽可能弱化数据质量对结果的影响, 能够更全面地反映 创新效率的变化, 是目前学者广泛采用的一种较为理想的非参数效率评价分析法。因此本文 采用非参数的 Malmquist 指数及其分解来测算创新效率 $A_{i t}$ 。

具体地，将每个省份看作为一个创新决策单位， $\mathrm{t}$ 时期的创新产出生产可能集定义为 $T^{t}=\left\{\left(x^{t}, y^{t}\right)\right\}$, 其中 $x^{t}$ 和 $y^{t}$ 分别表示 $\mathrm{t}$ 时期的创新投入向量和产出向量, 并且定义创新产出距 离函数 $D^{t}\left(y^{t}, x^{t}\right)=\inf \left\{\phi \succ 0:\left(x^{t}, y^{t} / \phi\right) \in T^{t}\right\}$, 其中 $\inf$ 表示集合的最大下界, 且 $D^{t}\left(y^{t}, x^{t}\right) \leq 1$ 当且仅 当 $y^{t}$ 处于 $x^{t}$ 决定的创新产出可能集的最前沿边界上时取 1 , 在此基础上可以构造出从 $\mathrm{t}$ 时期 到 $\mathrm{t}+1$ 时期 Malmquist 生产率指数:

$$
M_{i}\left(x^{t}, y^{t}, x^{t+1}, y^{t+1}\right)=\frac{D_{i}^{t+1}\left(x^{t+1}, y^{t+1}\right)}{D_{i}^{t}\left(x^{t}, y^{t}\right)}\left[\frac{D_{i}^{t}\left(x^{t+1}, y^{t+1}\right)}{D_{i}^{t+1}\left(x^{t+1}, y^{t+1}\right)} \times \frac{D_{i}^{t}\left(x^{t}, y^{t}\right)}{D_{i}^{t+1}\left(x^{t}, y^{t}\right)}\right]^{1 / 2}=E F F C H \times T E C H
$$

以 1 为界限, Malmquist 指数表明从 $\mathrm{t}$ 期到 $\mathrm{t}+\mathrm{I}$ 期的创新效率情况, 超过 1 , 表明提高了, 等于或小于 1 说明不变或下降, 其中 $\mathrm{EFFCH}$ 是技术效率的变化指数, 反映了决策单元对最佳 实践的追赶程度; $\mathrm{TECH}$ 是技术进步的变化指数, 反映了从两个时期生产前沿面的移动幅度, 它表示了技术进步的变化程度。

本文运用 Malmquist 指数测算高技术产业创新效率时，参考已有的文献的基础上，以利 用工业品出厂价格指数平减后的新产品销售收入、专利申请数作为创新的产出变量, 研发人 力投入变量、研发资本投入变量分别选择 $R \& D$ 人员全时当量以及 $R \& D$ 资本存量, 其中 $R \& D$ 资本存量永续盘存法估算公式:

$$
K_{t}=E_{t-1}+(1-\delta) \times K_{t-1}
$$

即 $\mathrm{t}$ 期的 $\mathrm{R} \& \mathrm{D}$ 资本存量（ $K_{t}$ ) 等于 $\mathrm{t}-1$ 期的 $\mathrm{R} \& \mathrm{D}$ 流量现值（ $E_{t-1}$ ） 与 $\mathrm{t}-1$ 期的 $\mathrm{R} \& \mathrm{D}$ 资本存 量 $\left(K_{t-1}\right)$ 之和。其中， $\delta$ 参考国际上的方法，作为恒定的折旧率 $15 \%$; 并参考李向东等 (2011) ${ }^{[1]}$ 的思路，将 R\&D 支出价格指数 (PR) 设定为:

$$
P R=0.75 \times P L+0.25 \times P I
$$

公式表示为消费价格指数 $(P L)$ 与固定资产投资价格指数 (PI) 的加权乘积, 0.75 和 0.25 分别是 2008-2013 年间劳务费和设备仪器支出占两者总和之比, 两者支出数据均来源于 R\&D 内部经费支出分类数据; 基期资本存量 $\left(K_{0}\right)$ 通过下式来估计:

$$
K_{0}=E_{0} /(g+\delta)
$$


$E_{0}$ 为基期实际 $\mathrm{R} \& \mathrm{D}$ 经费流量支出， $g$ 、 $\delta$ 分别为 2008-2013 年实际 $\mathrm{R} \& \mathrm{D}$ 经费支出的平 均增长率和折旧率。

3.2 .2 核心解释变量金融集聚水平的测算

区位熵可以衡量地方的产业专业化程度, 是衡量集聚水平的常用指标。其计算方法如下:

$$
\beta_{i j}=\frac{q_{i j} \div q_{j}}{q_{i} \div q}
$$

其中， $q_{i j} 、 q_{j}$ 分别表示地区 $\mathrm{j}$ 的 $\mathrm{i}$ 产业产值和全部工业产值， $q_{i} 、 q$ 分别是全国 $\mathrm{i}$ 产业总 产值和所有工业总产值。若该指数越大于 1 , 可以认为该产业是地区的专业化部门, 集聚化 程度也越高, 若指数小于或等于 1 , 则认为在该地区集聚效应不是很明显。本文借鉴丁艺等 （2010） [1]、洪功翔等（2014） [1]以银行业、证券业、保险业作为金融业的代表，将集聚区位 熵划分为相应的三个部分。

3.2.3 控制变量的测算

借鉴高技术产业效率研究的相关文献，本文加入研发经费强度（r）、研发人力资本强度 $(\mathrm{p})$ 、企业规模 $(\mathrm{t})$ 、市场集中度 $(\mathrm{m})$ 控制变量, 其中, 研发经费强度 $(r)$ 以 R\&D 研发 经费内部支出与主营业务收入之比表示; 研发人力资本强度 $(p)$ 以 R\&D 人员折合全时当量 与高技术产业就业人数之比表示; 企业规模 (t) 用地区企业平均规模即主营业务收入与企业 数之比表示; 市场集中度 $(\mathrm{m})$ 用地区企业数在全国的占比表示。各变量的描述和度量见表 1 。

表 1 变量描述和度量

\begin{tabular}{|c|c|c|}
\hline \multirow{3}{*}{ 被解释变量 } & tfp & 总创新效率, 用 Malmquist 指数测算 \\
\hline & eff & 技术效率变化指数, Malmquist 指数的分解 \\
\hline & tech & 技术进步指数, Malmquist 指数的分解 \\
\hline \multirow{3}{*}{ 解释变量 } & $\mathrm{b}$ & $\begin{array}{c}\text { 银行业区位熵, 各省银行存贷款总额占全国比重/各省 } \\
\text { 人口数占全国的比重 }\end{array}$ \\
\hline & $\mathrm{s}$ & $\begin{array}{c}\text { 证券区位熵, 各省发行总股本数占全国比重/各省人口 } \\
\text { 数占全国的比重 }\end{array}$ \\
\hline & $\mathrm{i}$ & $\begin{array}{c}\text { 保险业区位熵, 各省保费收入占全国的比重/各省人口 } \\
\text { 数占全国的比重 }\end{array}$ \\
\hline \multirow{4}{*}{ 控制变量 } & $\mathrm{r}$ & $\begin{array}{c}\text { 研发经费强度, R\&D 研发经费内部支出与主营业务收 } \\
\text { 入之比 }\end{array}$ \\
\hline & $\mathrm{p}$ & $\begin{array}{c}\text { 研发人力资本强度, R\&D 人员折合全时当量与高技术 } \\
\text { 产业就业人数之比 }\end{array}$ \\
\hline & $\mathrm{t}$ & 平均企业规模, 主营业务收入与企业数之比 \\
\hline & $\mathrm{m}$ & 市场集中度, 地区企业数在全国的占比 \\
\hline
\end{tabular}

\section{3 数据来源及处理}

本文面板数据的时间跨度是 2008-2013 年，地区跨度是 28 个省市（由于数据的缺失， 本文未包括内蒙古、西藏、青海、香港、台湾和澳门），其中，有关高技术产业的数据主要 来源于《中国高技术产业统计年鉴：2009-2014》，以及中华人民共和国科技部中国科技统 计网站的相关数据, 金融相关数据主要来源于 《中国金融统计年鉴：2009-2014》, 以及中 华人民共和国统计局官网的相关数据。

\section{4. 实证结果与分析}

\section{1 创新效率变化的计算结果说明}

选取 2008-2013 年全国 28 个省市的高技术产业相关数据, 运用 DEAP2.1 软件中的投入 导向 DEA 模型, 估算出每个省份的高技术产业的全要素生产率增长率 Malmquist 指数(TFP) 及其分解, 其中 Malmquist 指数结果如下所示。 
表 2 2009-2013 年全国 28 个省市 Malmquist 指数

\begin{tabular}{|c|c|c|c|c|c|c|c|}
\hline 地区 & & $2008-2009$ & $2009-2010$ & $2010-2011$ & $2011-2012$ & $2012-2013$ & 平均值 \\
\hline 北 & 京 & 0.708 & 0.956 & 0.908 & 1.171 & 0.809 & 0.898 \\
\hline 天 & 津 & 0.861 & 0.83 & 0.828 & 1.252 & 1.242 & 0.984 \\
\hline 河 & 北 & 0.888 & 0.776 & 1.221 & 1.252 & 1.086 & 1.027 \\
\hline 山 & 西 & 0.462 & 1.248 & 0.983 & 1.093 & 0.722 & 0.851 \\
\hline 辽 & 宁 & 0.817 & 0.806 & 1.142 & 0.808 & 1.267 & 0.949 \\
\hline 吉 & 林 & 1.818 & 0.381 & 2.088 & 0.937 & 0.991 & 1.061 \\
\hline 黑 龙 & 江 & 0.902 & 0.86 & 1.693 & 1.244 & 0.947 & 1.091 \\
\hline 上 & 海 & 0.446 & 0.823 & 1.172 & 0.953 & 0.946 & 0.827 \\
\hline 江 & 苏 & 0.59 & 0.842 & 1.577 & 1.01 & 0.905 & 0.935 \\
\hline 浙 & 江 & 1.07 & 0.689 & 1.458 & 1.099 & 1.073 & 1.048 \\
\hline 安 & 徽 & 2.359 & 0.704 & 1.777 & 0.987 & 0.939 & 1.223 \\
\hline 福 & 建 & 0.683 & 0.874 & 0.865 & 0.9 & 0.899 & 0.84 \\
\hline 江 & 西 & 1.206 & 0.945 & 1.103 & 1.175 & 1.281 & 1.136 \\
\hline 山 & 东 & 0.973 & 0.973 & 0.988 & 0.938 & 0.885 & 0.951 \\
\hline 河 & 南 & 1.414 & 0.86 & 1.243 & 0.935 & 3.302 & 1.361 \\
\hline 湖 & 北 & 1.734 & 0.709 & 1.263 & 1.005 & 0.927 & 1.077 \\
\hline 湖 & 南 & 2.167 & 0.675 & 2.136 & 0.818 & 1.288 & 1.269 \\
\hline 广 & 东 & 1.163 & 0.856 & 1.187 & 0.935 & 1.049 & 1.03 \\
\hline 广 & 西 & 1.338 & 0.622 & 1.9 & 0.764 & 1.289 & 1.093 \\
\hline 海 & 南 & 8.487 & 0.366 & 2.564 & 1.05 & 0.745 & 1.442 \\
\hline 重 & 庆 & 1.355 & 0.718 & 1.947 & 0.628 & 1.028 & 1.041 \\
\hline 四 & 川 & 1.331 & 0.661 & 2.142 & 0.936 & 0.818 & 1.076 \\
\hline 贵 & 州 & 1.34 & 0.662 & 1.002 & 1.08 & 0.87 & 0.965 \\
\hline 云 & 南 & 1.403 & 0.507 & 1.54 & 0.831 & 0.783 & 0.935 \\
\hline 陕 & 西 & 0.653 & 0.892 & 1.459 & 0.912 & 1.084 & 0.966 \\
\hline 甘 & 肃 & 1.205 & 1.521 & 0.942 & 1.477 & 0.816 & 1.158 \\
\hline 宁 & 夏 & 0.975 & 1.217 & 1.823 & 1.106 & 1.423 & 1.277 \\
\hline 新 & 疆 & 0.812 & 0.126 & 1.064 & 0.126 & 8.833 & 0.656 \\
\hline 平 均 & 值 & 1.124 & 0.729 & 1.36 & 0.925 & 1.114 & 1.028 \\
\hline
\end{tabular}

从表 2 可以看出，2008-2013 年全国高技术产业的创新效率 Malmquist 指数为 $1.028 ，$ 年 平均增长 $2.8 \%$, 体现出我国高技术产业创新效率总体上升的趋势; 并且在考察时段内, 全国 28 个省市的创新效率指数差异明显, 其中 16 个省市创新效率指数大于 1 即年平均正增长, 12 个省市指数小于 1 , 创新效率年均下降。

\section{2 金融集聚与高技术产业创新效率实证分析}

本文利用以上所得数据, 采用 2009-2013 年我国 28 个省份的面板数据, 运用 Eviews 6.0 软件分别以 $T F P$ 以及其分解指数 $E F F 、 T E C H$ 为被解释变量对金融集聚与我国高技术产业创 新效率的关系进行计量分析, 由于上文构建的计量模型有混合效应模型、固定效应模型以及 随机效应模型之分, 为了避免模型设定偏差引起的参数估计的有效性, 需要对模型的设定形 式进行检验。在计量分析上, 判断是采用混合模型还是个体固定效应模型一般通过构造统计 量 $F$ 来检验, 而常用 Hausman 检验来进行判别固定效应模型还是随机效应模型更有效, 具体 检验结果见表 3 , 结果表明, 全部 P 值均小于 0.05 , 因此拒绝原假设, 选择备选假设, 即本 文三个模型均选择固定效应模型。 
表 3 面板模型类型选择检验

\begin{tabular}{c|cccc}
\hline & & TFP & EFF & TECH \\
\hline $\mathrm{F}$ 检验 & 统计量 & 2.47188 & 3.630378 & 7.467029 \\
& $\mathrm{P}$ 值 & 0.0006 & 0.0000 & 0.0000 \\
\hline Hausman 检 & 统计量 & 14.933511 & 50.123781 & 97.721784 \\
& $\mathrm{P}$ 值 & 0.0369 & 0.0000 & 0.0000 \\
\hline 模型形式 & & 固定模型 & 固定模型 & 固定模型 \\
\hline
\end{tabular}

另外, 采用面板数据的个体固定效应模型对全国范围内进行估计时, 截面个数（28）远 大于时序个数 (5), 为防止异方差, 在估计中采用截面加权的广义最小二乘估计（GLS）。 回归结果如表 4 所示。

表 4 模型回归结果

\begin{tabular}{|c|c|c|c|}
\hline 变量 & (1) TFP & (2) $\mathrm{EFF}$ & (3) TECH \\
\hline $\mathrm{C}$ & -0.772006 & 1.327352 & $-2.777603 * *$ \\
\hline $\operatorname{lnb}$ & $1.075672 * * *$ & $-0.866695 *$ & $2.830602 * * *$ \\
\hline $\operatorname{lns}$ & $0.320941 * *$ & $-0.496283^{*}$ & $0.789999 * * *$ \\
\hline $\ln i$ & -0.515995 & $-1.104322 *$ & 0.550325 \\
\hline $\ln r$ & $0.601766^{* * * *}$ & 0.070145 & $0.518989 * * *$ \\
\hline $\ln p$ & $-0.424992 * * *$ & 0.126939 & $-0.406257 * * *$ \\
\hline $\ln t$ & $0.172715^{* *}$ & $-1.249960 * * *$ & $1.179579 * * *$ \\
\hline $\operatorname{lnm}$ & $-0.538965 * *$ & -0.013732 & $-0.848312 * * *$ \\
\hline F 统计值 & $2.729728 * * *$ & $7.872120 * * *$ & $14.06715^{* * * *}$ \\
\hline 样本数 & 140 & 140 & 140 \\
\hline
\end{tabular}

注: ***、**、*分别表示在显著水平 $1 \% 、 5 \% 、 10 \%$ 下显著。

从总创新效率 (TFP) 层面分析，模型（1）的回归结果显示银行业集聚的弹性系数为 1.075672 , 在 $1 \%$ 显著水平下显著, 证券业集聚的弹性系数为 0.320941 , 在 $5 \%$ 显著水平下显 著, 而保险业集聚未能通过显著性检验，我们可以得出，银行业集聚与证券业集聚与我国高 技术产业总创新效率（TFP）显著地正相关, 即两者能显著地促进总创新效率的提高, 并且 我国银行业集聚相对于证券业集聚对高技术产业创新效率的影响更大，而保险业集聚对我国 高技术产业总创新效率的影响不明显，这是因为我国金融市场结构以银行为主导，从而银行 部门相对于证券市场和保险市场对经济的影响程度大很多; 此外, 研发经费投入强度、平均 企业规模对总创新效率有显著正效应，企业集中度、研发人员投入强度有显著负效应。

为了进一步分析金融集聚对总创新效率的影响途径，把总效率指数（TFP）分解为技术 进步指数 (TECH) 和技术效率指数 $(\mathrm{EFF})$, 分别作为被解释变量进行回归分析, 其中 TECH 即模型（2）的回归结果显示银行业集聚与证券业集聚能显著地促进高技术产业技术进步的增 长，保险业集聚效应不明显，控制变量中研发经费投入强度、平均企业规模对总创新效率有 显著正效应，企业集中度、研发人员投入强度有显著负效应，该模型各变量系数回归结果与 总效率模型所得完全一致, 并且通过比较两模型各回归系数大小可以看出, 各变量对 TECH 的影响要大于对 TFP 的影响; 而在 EFF 即模型（3）中，银行业集聚、证券业集聚和保险业 集聚的弹性系数均在 $10 \%$ 的显著性水平下为负值，即金融集聚对高技术产业技术效率产生了 较为显著的负影响, 这是因为中国高技术产业发展还未成熟并处于发展中国家的大环境中, 
而根据 Meon and Weill（2010） ${ }^{[1]}$ 的研究，金融发展的技术效率增长效应在发展中国家会比较 微弱, 甚至会出现负影响, 并且金融规模的无效率扩张不利于技术创新和制度创新, 进而间 接阻碍了 TFP 增长（谢朝华等，2014） [1]，这说明金融集聚对高技术产业创新效率影响效应 的驱动力主要来自金融集聚的高技术产业技术进步效应，而金融集聚带来的技术效率负效应 在相当程度上减弱了金融集聚对技术进步效应的效果。

\section{5. 结束语}

本文利用 2008-2013 年间省级层面的面板数据, 在 DEA-Malmquist 指数方法的基础上 利用面板模型对金融集聚与我国高技术产业自主创新效率间的本质关系进行探讨, 结果发现, 除了保险业集聚效应不显著，金融集聚（银行业集聚、证券业集聚）能显著地促进高技术产 业创新效率，并且这一效应的驱动来源主要是技术进步效应，而不是技术效率效应。

根据以上所得结论，本文得到如下政策建议：第一，提高产业自主创新水平是重点。高 技术产业的发展不能一味地追求规模效应, 更需要对产业自身的技术进步水平加大关注, 只 有技术进步水平上升和技术效率提高两者同时得到重视, 高技术产业才能健康地发展。第二, 不断扩大金融集聚对地域的辐射效应。首先，要因地制宜，针对各地区的特色推出差异化金 融产品, 并且由于在实证中结果显示银行市场对高技术产业创新效率有最为显著的作用, 所 以要继续优化银行业结构, 继续发挥银行业的主导促进作用, 其次, 构建多层次的、完善的 证券市场，促使高技术企业研发资金的募集可以在证券市场按需完成，从而加大证券业集聚 对高技术产业创新效率的促进作用，最后，由于在实证中保险集聚对高技术产业创新效率的 作用不明显, 所以要加大监管力度以及不断完善保险市场的目标定位, 从而发挥其优势，不 断将保险业集聚效应的潜力发挥出来。第三，加深金融业市场化，提高金融的集聚效率水平。 因为在市场化的过程中，金融资源能更加合理地流动，金融集聚现象是由市场本质需求出发 来决定，从而就能不断提高金融集聚的质量，不断降低甚至扭转金融集聚对高技术产业技术 效率的负效应，进而促进总创新效率的提高。

\section{References}

[1] Schumpeter J A. The theory of economy development [M]. Cambrige, MA: Harvard Univerisity Press, 1912:54-56 .

[2] Hicks J A. Theory of economic history [M]. Oxford: Claredon Press, 1969.

[3] Romer P. Endogenous Technological Change [J]. Journal of Political Economy, 1990, 98(5):71-102.

[4] Saint-Paul G. Technology Choice, Financial Markets and Economic Development [J]. European Review, 1992,36(4):763-781.

[5] Luigi Benfratello, Fabio Schiantarelli and Alessandro Sembenelli. Banks and innovation:Micro Econometric Evidence on Italian Firms [J]. Journal of Financial Economics, 2008,(90):197-217.

[6] Brown, J. R., Fizzers, D. M., Petersen, B. C. Financing Innovation and Growth: Cash Flow, External Equity, and the 1990S R\&D Boom [J]. Journal of Finance 2009, (64):151-185.

[7] Ba Tusong.The Financial Support The Development Of High-Techindustry Industry In China [J].Urban Finance Forum , 2000, 5(1):15-21.

[8] DENG Nan. Financial Supporting System for the High-tech Industry Development in China[J]. China Soft Scinence, 1999(10):9-13.

[9] Ma Xiaoxia, Research on Financial Support System of High-tech Industry[J]. Science \& Technology Progress And Policy, 2006, 23(9):88-90. 
[10]Huang Dechun Min Zunxiang Xu Min. Financial Development and Technological Innovation:An Empirical Research of Chinese New High-tech Industry[J]. Forum on Science and Technology in China, 2011, 17(12):21-25.

[11]Zhang Changzheng,Huang Dechun ,Ma Zhaojie. Industrial Agglomeration and Industrial Innovation Efficiency:Coupling Effects of the Financial Markets-Based on the Agglomeration and Innovation of China Hi-tech Industry[J]. Industrial Economics Research, 2012(6):17-25.

[12] Ma Yanxin, Financial Support and Innovation of Chinese High-tech Industry:Panel Data Model Study[J]. Journal of Guangxi Financial Research, 2012(1):20-27.

[13]Liu Jun, Huang Jieyu, Cao Lijun, The System of Financial Agglomeration To The Economic [J]. Management World, 2007(4):152-153.

[14]Ding Yi, Li Lin, Li Bin. The research of the Relation of the Financial Agglomeration and Local Economic growth [J]. Statistics And Decision, 2009(6):131-134.

[15] Shi Pei Pu Yongjian. Analysis on Spatial Correlation Mechanism between Financial Agglomeration and Industrial Structure [J].Technology Economics, 2011, 30(1):39-44.

[16] Sun Jin, Li Hansuo. Financial Agglomeration and Industry Improvement-Based on the data of 2003-2007 [J].Economist, 2012(3):80-86.

[17]Pan Hui, Rang Guanghe, Zhang Bin. The research of the arears' Difference of the Relation of the Financial Agglomeration and Economic growth[J]. Inquiry Into Economic Issues , 2013(5):102-107.

[18]LI Xiang-dong, LI Nan, BAI Jun-hong, XIE Zhong-qiu. Study on the R\&D Innovation Efficiency of High - tech Industry in China [J]. China Soft Scinence, 2011(2):52-61.

[19]Ding Yi, Li Jingxia, Li Lin. Financial Agglomeration and Economic growth-based on the provincial data [J]. Insurance Research, 2010(2):20-30.

[20]HONG Gong-xiang, ZHANG Lan-ting,LI Wei-jun. Regional Heterogeneity of the Effect of Financial Agglomeration on Total Factor Productivity-An Empirical Study Based on Dynamic Panel Data Model [J]. Economic Survey, 2014, 31(4):7-12.

[21]Méon P G, Weill L. Does financial intermediation matter for macroeconomic performance?[J]. Economic Modelling, 2010, 27(1):296-303.

[22]XIE Chao-hua LIU Heng-sha. A Empirical Study on the Association Mechanism Between Financial Development and TFP Growth in China Based on the Mediation Effect of the Innovation of Technology and System [J]. The Theory and Practice of Finance and Economics, 2014, 35(1):33-38. 